\title{
The stereo-PIV investigation of the unsteady flow in the draft tube of a model hydro turbine
}

\author{
I. Litvinov ${ }^{1,2 *}$, D. Sharaborin ${ }^{1,2}$, S. Shtork ${ }^{1}, 2$, V. Dulin ${ }^{1,2}$, \\ S. Alekseenko ${ }^{1,2}$, and Kilian Oberleithner ${ }^{3}$ \\ ${ }^{1}$ Kutateladze Institute of Thermophysics, Novosibirsk, Russia \\ 2 Novosibirsk State University, Novosibirsk, Russia \\ ${ }^{3}$ Technische Universität Berlin, Berlin, Germany \\ *litvinov@itp.nsc.ru
}

\begin{abstract}
Varying the generator load of a hydro turbine results in short-term changes in the rotation frequency of the runner, leading inevitably to flow instability and strong flow swirling behind the turbine. This may lead to the formation of unsteady flow regimes featured by vortex instability of the swirling flow behind the runner, known as the precessing vortex core (PVC) Dörfler et al. (2012). This effect causes dangerous periodic pressure pulsations that propagate throughout the water column in the draft tube. The present study reports on stereo PIV measurements of the air flow field inside a transparent draft tube of a model hydro turbine for a wide range of operation conditions. The research is focused on the time-averaged flow properties (mean velocity field and the second-order moments of velocity fluctuations), pressure pulsations and coherent flow structures in the velocity field.

A detailed description of the aerodynamic test rig, used in these experiments, is presented in Litvinov et al. (2018). The test section consists of a scaled-down geometric model of the Francis-99 draft tube Cervantes et al. (2015) with an inlet diameter $D=100 \mathrm{~mm}$. The air flow inside the model is investigated. To generate the required flow distribution at the inlet to the cone, a pair of swirlers is used: a stationary swirler, acting as guide vanes, and a rotary swirler, representing an analogue of the turbine runner Litvinov et al. (2018). The pair of swirlers is designed for the optimal operating conditions (the best efficiency point - BEP) corresponding to a volumetric flow rate $Q_{c}=48.5 \mathrm{l} / \mathrm{s}$ and a runner rotation speed $n_{c}=40.5 \mathrm{~Hz}$. The experimental setup includes a computer for controlling the airflow and rotation frequency of the rotor with an uncertainty of $1.5 \%$ and $0.5 \%$, respectively. The flow is studied for the operating parameters of the flow rate in the range from $0.5 Q_{c}$ to $Q_{c}$ to replicate the off-design and the BEP operation conditions of the hydro turbine.

Figure 1 shows the used PIV equipment and a sketch of the test section. A Laskin nozzle aerosol generator was used to seed the jet flow with seed oil droplets. A double-head Nd:YAG laser (Quantel, EverGreen) illuminated the tracer particles. The laser beam was converted into a laser sheet with a thickness of less than $1 \mathrm{~mm}$ by using a system of cylindrical and spherical lenses. On average, the pulses energy was $70 \mathrm{~mJ}$ before the laser sheet optics (measured by a power meter Coherent LabMax). Particles images were captured by a pair of CCD cameras (Bobcat ImperX). The PIV images (4904x3280 pixels is size) were processed by using an in-house software ActualFlow. Time separation between two PIV laser pulses was $30 \mu \mathrm{s}$. The images were preprocessed to remove background (minimal intensity for each pixel). During four iterations of an adaptive cross-correlation algorithm the interrogation area size was reduced from $64 \times 64$ to $16 \times 16$ pixels. The spatial overlap rate between the interrogation areas was $50 \%$, and the resulting spatial resolution was $0.5 \mathrm{~mm}$

Four Behringer ECM8000 microphones were used to record the pressure pulsations on the draft tube walls in the cross-section A-A as shown in Figure 1. Microphone signals were digitized by an ADC and amplified using Behringer preamplifiers. The PVC frequency was identified via time-resolved pressure signals at a sampling rate of $20 \mathrm{kHz}$. The microphone signals were decomposed into azimuthal spatial and time Fourier domains. Figure 2 shows an example of the power spectral density of the asynchronous part of the pressure pulsations $p^{a s, i}(t)=p^{i}(t)-(1 / 4) \sum_{i=1}^{4} p^{i}(t)$ for the cases of flow rates $0.5 Q_{c}$ (off-design) and $Q_{c}$ (BEP regime). As shown, the most energetic peak in the spectra occurs for the case of $0.5 Q_{c}$. This dominant
\end{abstract}



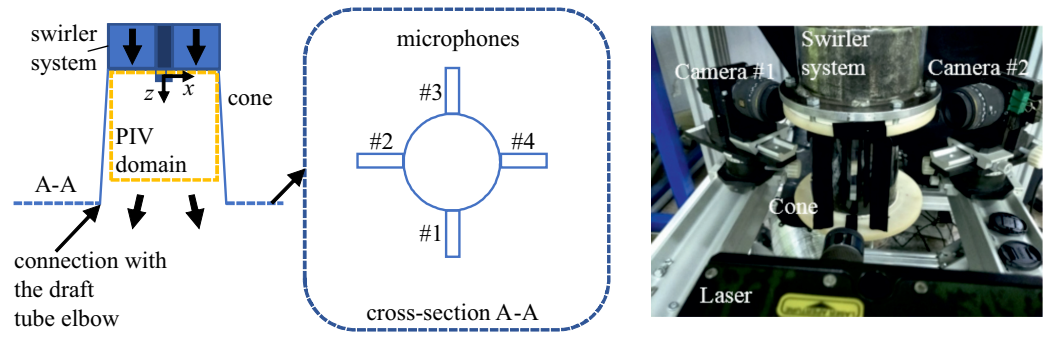

Figure 1: 2D sketch of the test section (left) and photo of the PIV configuration (right)

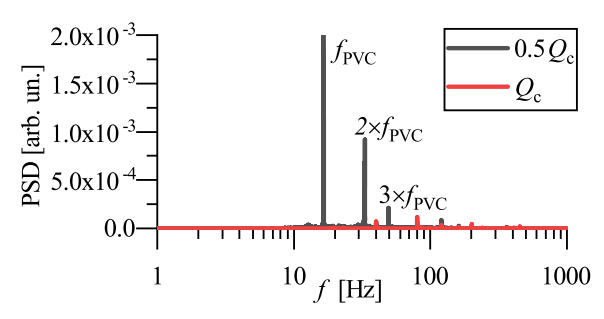

Figure 2: Spectra of the asynchronous part of the pressure pulsation of four acoustic sensors (the vertical axis in relative units) for a set of flow rates $0.5 Q_{c}$ (off-design) and $Q_{c}(\mathrm{BEP}$ regime)

peak is considered to be associated with the PVC. Figure 3 shows the mean velocity field for the flow rates $0.5 Q_{c}$ (off-design) and $Q_{c}$ (BEP regime). For the regime with $0.5 Q_{c}$, when the flow dynamics is related to strong pulsations due to the PVC, the time-averaged velocity field is characterized by a compact recirculation zone with the length of $0.2 D$. For the BEP regime with $Q_{c}$ is characterized by a smaller recirculation region $(0.1 D)$, which is associated with the flow past the centrebody. It is noteworthy that this flow is featured by the flow core rotation in the opposite direction to the sense of the main flow swirl. Further analysis is focused on the phase-averaged velocity fields and on the results of proper orthogonal decomposition (POD).

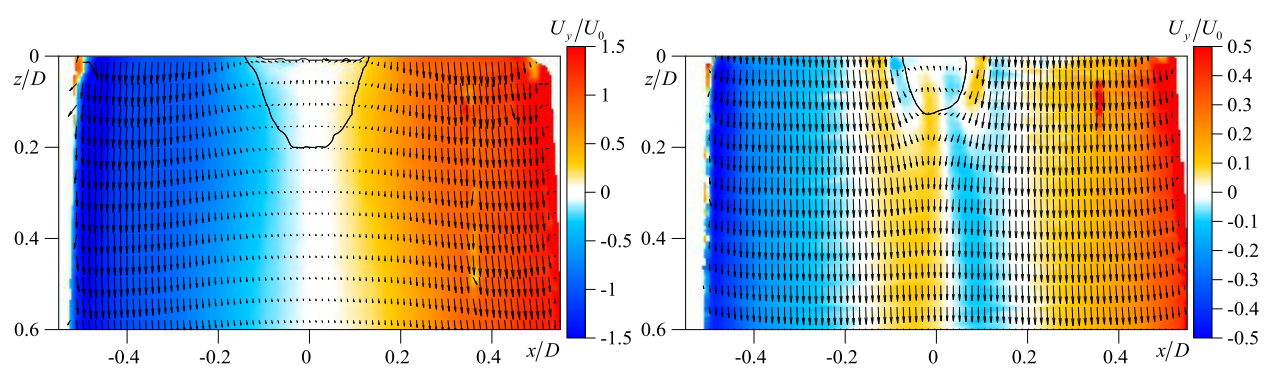

Figure 3: Mean velocity fields for the $0.5 Q_{c}$ case, the off-design condition with PVC (left) and the BEP regime, $Q_{c}$ (right), $\left(U_{0}\right.$ is a bulk velocity, a solid line indicates recirculation region border)

\section{Acknowledgements}

The research is supported by Russian Foundation for Basic Research (Grant no. 20-58-12012). I. Litvinov acknowledges support of the grant of the President of the Russian Federation (project no. MK-1504.2021.4, in the part of pressure signal analysis). The funding of the German Research Foundation (grant no. 429772199) is acknowledged.

\section{References}

Cervantes M, Trivedi C, Dahlhaug OG, and Nielsen T (2015) Francis-99 workshop 1: steady operation of francis turbines. in J. Phys.: Conf. Ser. volume 579. page 011001

Dörfler P, Sick M, and Coutu A (2012) Flow-induced pulsation and vibration in hydroelectric machinery: engineer's guidebook for planning, design and troubleshooting. Springer Science \& Business Media

Litvinov I, Shtork S, Gorelikov E, Mitryakov A, and Hanjalic K (2018) Unsteady regimes and pressure pulsations in draft tube of a model hydro turbine in a range of off-design conditions. Experimental Thermal and Fluid Science 91:410-422 